\title{
Adaptation and reliability testing of the SHARE-FI instrument for the assessment of risk of frailty syndrome among older Polish patients
}

\author{
MARTA MUSZALIK ${ }^{1, \text { A-F }}$, EWA BOROWIAK ${ }^{2, \text { в, D-F }}$, AGNIESZKA KOTARBA ${ }^{2, \text { B, D-F}}$, GRAŻYNA PUTO ${ }^{3, \text { B, D-F }}$, \\ HALINA DOROSZKIEWICZ ${ }^{4, B, D-F}$, KORNELIA KĘDZIORA-KORNATOWSKA ${ }^{1, A, D}$ \\ ${ }^{1}$ Chair and Clinic of Geriatrics of Nicolaus Copernicus University Collegium Medicum in Bydgoszcz, Poland \\ ${ }^{2}$ Department of Nursing Teaching Medical University in Lodz, Poland \\ ${ }^{3}$ Jagiellonian University Medical College Faculty of Health Sciences Nursing and Obstetrics Institute Clinical Nursing \\ Unit in Cracow, Poland \\ ${ }^{4}$ Department of Geriatrics, Medical University of Bialystok, Poland
}

A - Study Design, B - Data Collection, C - Statistical Analysis, D - Data Interpretation, E - Manuscript Preparation, F - Literature Search, G - Funds Collection

Summary Background. Frailty reduces independence, quality of life and psychological well-being. Frailty also increases the risk of geriatric syndromes, addictions, hospitalization, institutionalization, disability and mortality in the oldest population of every society. Objectives. The main aim of this research was the adaptation and validation of the SHARE-FI questionnaire, identifying the risk of frailty syndrome in a group of people over 60 years of age in Poland. Another aim was to create Polish calculators for the SHARE-FI questionnaire for females and males separately.

Material and methods. Testing with the SHARE-FI questionnaire was performed on 300 people over 60 years of age in Poland. The study group consisted of 148 females and 152 males, including 151 hospital and 149 primary care patients. The mean age was 75.2. Results. Cronbach's alpha reliability coefficients of the SHARE-FI instrument ranged from 0.73 to 0.83 , and item-total correlation ranged from 0.11 to 0.91 . The risk of frailty syndrome was significantly higher in the group of hospital patients than in the group of primary care patients $(p<0.001)$. The average score on the IADL scale was 23.09 for the study group, while the GDS score indicated no depression in 203 patients and mild depression in 97 patients.

Conclusions. The research results indicated that the Polish version of the SHARE-FI questionnaire is characterized by high internal consistency and reliability and may be recommended for the screening frailty risk among people above 60 years of age for females and males, as well as in both primary care and hospital settings.

Key words: frailty, geriatric assessment, primary health care, mass screening, validation studies.

Muszalik M, Borowiak E, Kotarba A, Puto G, Doroszkiewicz H, Kędziora-Kornatowska K. Adaptation and reliability testing of the SHARE-FI instrument for the assessment of risk of frailty syndrome among older Polish patients. Fam Med Prim Care Rev 2018; 20(1): 36-40, doi: https://doi.org/10.5114/fmpcr.2018.73702.

\section{Background}

The priority of geriatric care in Poland and worldwide is to maintain maximum fitness and independence in life, provide treatment and rehabilitation, enhance health-related quality of life, as well as prevent unnecessary institutionalization [1-3]. According to experts from the World Health Organization, an indicator of the lack of favorable aging is geriatric frailty [4]. Geriatric frailty is associated with long-term health complications, increased risk of geriatric syndromes, addictions, hospitalization, institutionalization, disability and mortality in the oldest population of every society [5-9].

Poland's demographic and epidemiological situation has prompted a change in the planning, organization and operation of health care. An essential aspect of this system's modification is for medical professionals to use appropriate, effective and simple assessment tools [9]. So far, no optimum method of frailty identification has been developed. Nevertheless, it should be emphasized that early recognition of the syndrome and initiation of appropriate preventive measures may significantly delay the moment of total loss of independence through improving an elderly and frail person's ability to function in his or her current environment [10-12]. Frailty reduces independence, quality of life and psychological well-being, as well as becomes a difficult challenge for families, caregivers and welfare services [13-15].

Many frailty definitions and measurement tools have been developed in clinical practice and comparative studies, but the greatest international recognition has been achieved by the frailty phenotype definition by Fried et al. [16].

Santos-Eggimann et al. considered Fried's criteria in the first European research on the incidence of frailty (Survey of Health, Ageing and Retirement in Europe (SHARE)), in a group of middle-aged (above 50) and older community-dwelling Europeans living in ten countries. The researchers distinguished five SHARE criteria. It was the first attempt at operationalizing Fried's frailty phenotype in a very large European population-based sample [17]. The incidence of frailty in European society (65 years and older) ranges from $5.8 \%$ to $27.3 \%$, whereas from $34.6 \%$ to $50.9 \%$ of the elderly adult population is considered 'pre-frail' [17].

The aging of the society and increasing costs of care have contributed to an endeavor to create a simple frailty risk assessment tool in the European population of elderly people. This was done through the adaptation of a clinical paradigm based on the frailty concept and Fried's criteria, consistent with the biopsychosocial model of primary care. The authors created and 
validated a simple instrument for frailty screening in a group of people above 50 years of age [18].

The SHARE Frailty Instrument (SHARE-FI) is mainly designed to facilitate quick frailty risk assessment in the elderly adult population in a primary care setting [19].

In recent years, research into this subject has been carried out in Europe. SHARE-Fl is characterized by a sufficient structure and predictive value. Another important feature is easy access and application by using Internet calculators. The SHARE-FI questionnaire has proven to be an effective diagnostic instrument of frailty syndrome among European senior citizens [20$-22]$. The creation of the SHARE-FI questionnaire was also the first European research effort aimed at reaching a consensus over frailty risk assessment studies.

Regrettably, the questionnaire's validity and reliability have not been analyzed among the Polish society so far.

\section{Objectives}

The aim of this research was the adaptation and validation of the SHARE-FI questionnaire, identifying the risk of frailty syndrome in a group of people over 60 years of age in Poland. Another aim was to create Polish calculators for the SHARE-FI questionnaire for females and males separately.

\section{Material and methods}

\section{Translation procedures}

The SHARE-FI questionnaire was received from Dr. Romero Ortuno, along with consent to its translation and exploration. The English version of the SHARE-FI questionnaire was translated by three independent translators specializing in medical sciences. Translated questionnaire items were discussed by a group of researchers involved in the adaptation process in order to achieve a high level of clarity. The process was then reversed, and the Polish version was translated into English by an expert translator. All item descriptions and criteria were subsequently adapted in such a way so that the highest level of lucidity could be achieved. The Polish version was also presented to physicians and nurses to evaluate its clarity and comprehensibility.

\section{Sample}

Testing with the SHARE-FI questionnaire was performed on 300 elderly people over 60 years of age in Poland. The study group consisted of 148 females and 152 males, including 151 hospital (internal and geriatrics wards) and 149 primary care patients. The mean age was 75.2. The study covered people over 60 years of age suffering from various chronic conditions: high blood pressure or hypertension, diabetes or high blood sugar, chronic lung disease, such as chronic bronchitis or emphysema, asthma, osteoporosis, rheumatic disease, stroke victims and others. The average number of conditions was 3 , while the average number of medicines taken was 5 . The study exclusion criteria were difficulty in communication with the patient due to poor hearing or severe cognitive impairment, severe depression and complete lack of manual dexterity.

The study was conducted between day 3 to day 5 of a patient's stay in the hospital. All patients were in good health condition, which was confirmed by a medical examination.

Patients from primary care had the same selection criteria as above and were also examined by a physician. The study was conducted after a visit to the doctor.

\section{Measurements and procedure}

Patient assessment was performed by nurses trained in carrying out examinations. The training included obtaining information about the examination procedure, vital assessment ele- ments and practical instructions on applying the questionnaire and measuring muscle strength.

The SHARE Frailty Instrument consists of 5 questions regarding:

1. Exhaustion - In the last month, have you had too little energy to do the things you wanted to do?

2. Loss of appetite - What has your appetite been like?

3. Weakness - Maximum grip strength in kilograms: Right hand, Left hand; Attempt 1, Attempt 2.

4. Walking difficulties - Because of a health or physical problems, do you have any difficulty doing any of the following everyday activities?

(Exclude any difficulties that you expect to last less than 3 months): Walking 100 meters; Climbing one flight of stairs without resting.

5. Low physical activity - How often do you engage in activities that require a low or moderate level of energy, such as gardening, cleaning the car or going on a walk?

The items contain a cafeteria-style list of answers placed in an automatic calculator. All answers to questions have to be considered in the questionnaire. Based on the answers provided and measurements performed, the calculator then classifies the patient as belonging to one of the 3 categories: frail, pre-frail and non-frail.

Additionally, the study applied the Geriatric Depression Scale (GDS) and Instrumental Activity of Daily Living (IADL) questionnaires.

The Geriatric Depression Scale (GDS) was created by Yesavage et al., to be subsequently tested and used in the population of elderly people, both among healthy individuals, as a screening test, and among ill individuals, as well as to examine people with mild to moderate cognitive impairment. The long, 30-item form of the questionnaire assesses the mental state of the respondent over the previous week. The GDS questions are answered with "yes" or "no" by the subject [23]. Shorter, 15- and 4-item versions of the questionnaire have been developed since 1986. The long and short GDS versions serve for the self-assessment of symptoms of depression in elderly people and achieve a high level of correlation in research $(r=0.84, p<0.001)$ [23]. GDS questionnaire assessment does not replace a medical examination and history-taking, but is useful for a quick screening assessment of the patient's mental state. The GDS questionnaire may also be applied for monitoring the patient's state in various settings. High assessment scores require thorough examination of the patient by a psychologist or psychiatrist. The authors' own study used the short, 15-item GDS version by Yesavage for assessing the emotional state of elderly people; scores from 0 to 5 points indicate normal state, from 6 to 15 points - depression of increasing severity [24].

For assessment of a range of complex activities of daily living, the Instrumental Activities of Daily Living (IADL) scale was used. The IADL scale assesses instrumental activities in 8 areas of functioning, i.e. ability to use the telephone, housekeeping, shopping, food preparation, mode of transportation, ability to handle finances and responsibility for own medications. The total score is relevant to a particular patient and a fall in the score on consecutive examinations reflects a deteriorated general state. The result of the activity of daily living assessment of an elderly person on this scale allows for the objectivizing of the patient's needs for care or necessary assistance [25].

Medical records and the socio-demographic data obtained were also analyzed.

Testing was carried out at the Geriatrics Clinic and outpatient clinics in Bydgoszcz, Cracow and Lodz in Poland. Permission to perform the test was provided by the Bioethical Commission of the Nicolaus Copernicus University in Torun and the Ludwik Rydygier Collegium Medicum in Bydgoszcz, the Medical University in Lodz and the Medical University in Cracow, Poland. All participating patients provided informed consent. Examination was voluntary, and the sample selection was random. 


\section{Data analysis}

A descriptive analysis of the research results obtained used tables showing numbers and percentages. The mean, along with the standard, deviation was also calculated. Correlations between two variables were computed using Spearman's rank correlation coefficient R. Cronbach's alpha was used for the analysis of internal consistency and reliability coefficients of the SHARE-FI instrument. The results were considered statistically significant if $p \leq 0.05$. All statistical analyses were performed with the Statistica 10.0 program.

\section{Results}

\section{Descriptive analyses}

A majority of the respondents were city-dwellers - 246 people $(82 \%)$, while the remaining $54(18 \%)$ were rural residents. The marital status of the respondents was as follows: $125(41.7 \%)$ were in a relationship, 34 (11.3\%) single, 141 (47\%) widowed. The self-reported financial situation was considered average by most respondents - 176 people $(58.9 \%)$, good by $69(23.1 \%)$, very good by $8(2.7 \%)$, and $46(15.4 \%)$ considered themselves to be living below the average standard. The average score on the IADL scale was $\mathbf{2 3 . 0 9}$ for the study group, while GDS scores indicated no depression in 203 people and a risk of depression or depression of increasing severity in 97 people.

\section{Reliability}

The reliability of the SHARE-FI was analyzed in terms of internal consistency using Cronbach's alpha. Cronbach's alpha, a widely used reliability index, was calculated the scale level for the entire sample. The research assessed the internal consistency of the SHARE-FI questionnaire, for which the overall Cronbach's alpha was 0.79 . Table 1 shows the results of reliability coefficients and average frailty incidence in patients in hospital and primary care settings.

Table 2 show the results of Cronbach's alpha reliability coefficients of the SHARE-FI instrument ranged from 0.73 to 0.83 and corrected item-total correlation ranged from 0.11 to 0.91 .

\begin{tabular}{l} 
Table 1. Characteristics of frailty according to SHARE-FI in the \\
study group \\
\hline
\end{tabular}

An attempt was also made at assessing the risk of frailty syndrome in the study group. Frailty syndrome incidence was significantly higher in the group of hospital patients than in the group of primary care patients $(p<0.001)$. Figure 1 shows the significant differences in percentages of respondents in primary care and hospital settings in terms of determining the frailty level $(p<0.001)$

Table 2. Analysis of the reliability of the Polish version of the SHRE-FI instrument (original and adapted versions)

\begin{tabular}{|l|l|l|}
\hline Variable & $\begin{array}{l}\text { Corrected } \\
\text { item - total } \\
\text { correlation }\end{array}$ & $\begin{array}{l}\text { Cronbach's } \\
\text { alpha if item } \\
\text { deleted }\end{array}$ \\
\hline Exhaustion & 0.20 & 0.83 \\
\hline Loss of appetite & 0.11 & 0.83 \\
\hline Walking difficulties & 0.19 & 0.83 \\
\hline $\begin{array}{l}\text { Climbing one flight of stairs with- } \\
\text { out resting }\end{array}$ & 0.15 & 0.83 \\
\hline Low physical activity & 0.27 & 0.83 \\
\hline Weakness: Right hand Attempt 1 & 0.88 & 0.74 \\
\hline Weakness: Right hand Attempt 2 & 0.91 & 0.73 \\
\hline Weakness: Left hand Attempt 1 & 0.91 & 0.73 \\
\hline Weakness: Left hand Attempt 2 & 0.90 & 0.74 \\
\hline
\end{tabular}

Cronbach's alpha $=0.79$.

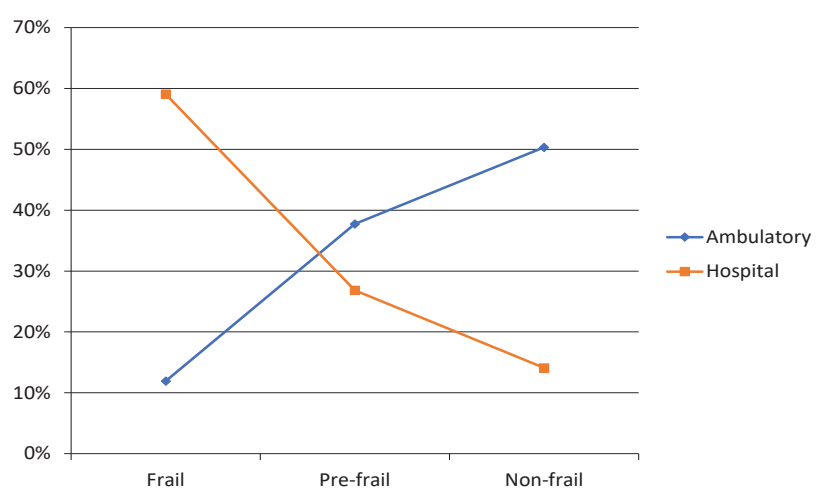

Figure 1. Prevalence of frailty risk in the study group (ambulatory and hospital patients)

Table 3 presents aggregate results of SHARE-FI questionnaire validation by gender. Statistically significant concordance was noted for all the questionnaire items, and the results were similar to those observed by the authors of the questionnaire [19].

\begin{tabular}{|c|c|c|c|c|c|c|c|c|}
\hline & \multicolumn{4}{|c|}{ Females } & \multicolumn{4}{|l|}{ Males } \\
\hline & $n=68$ & $n=68$ & $n=67$ & & $n=38$ & $n=29$ & $n=30$ & \\
\hline Frailty items & Frail & Pre-frail & Non-frail & $p$ & Frail & Pre-frail & Non-frail & $p$ \\
\hline Exhaustion: yes (cp) & 0.88 & 0.46 & 0.58 & 0.001 & 0.95 & 0.66 & 0.37 & 0.001 \\
\hline Weight loss: yes (cp) & 0.54 & 0.18 & 0.24 & 0.001 & 0.66 & 0.21 & 0.03 & 0.001 \\
\hline $\begin{array}{l}\text { Low activity: hardly ever, } \\
\text { or never (cp) }\end{array}$ & 0.91 & 0.57 & 0.58 & 0.001 & 0.68 & 0.24 & 0.03 & 0.001 \\
\hline Slowness: yes (cp) & 0.51 & 0.26 & 0.36 & 0.05 & 0.61 & 0.55 & 0.17 & 0.005 \\
\hline Handgrip strength: mean (Kg) & 14.54 & 18.93 & 18.18 & 0.002 & 25.37 & 28.93 & 34.67 & 0.001 \\
\hline
\end{tabular}

cp: conditional probability (from the DFactor model profile output). 
Having followed the above-described research procedure, two calculators were created in HTML format for females and males separately.

\section{Discussion}

Frailty as a physiological and multidimensional syndrome characterized by falling reserves and lowered resistance to stress-inducing factors, which is significantly connected with an increased risk of losing independence, frequent hospitalization, susceptibility to falling ill more often or even premature death. Numerous efforts have been made to offer unambiguous frailty definitions and diagnostic criteria [26]. In practice, one of the most commonly used diagnostic tools is the criteria proposed by Fried et al. [16]. No fully effective frailty syndrome treatment has been developed so far. However, the prophylactic role of preventing the effects of frailty and complications has been emphasized [27]. Early frailty recognition, along with instituting individually selected and directed preventive measures, may significantly delay the moment of losing independence and becoming reliant upon help. This also affects an improved quality of life in a home setting. Undiagnosed frailty problems may cause unfavorable aging, with the resultant necessity of institutional care. Frailty may co-occur with disability and multimorbidity; hence, early diagnosis may be even more useful in organizing care [28]. The aim of this research was the intercultural adaptation and validation of the SHARE-FI questionnaire, identifying the risk of frailty syndrome in a group of people over 60 years of age in a study group, as well as creating Polish calculators for the SHARE-FI questionnaire for females and males separately.

The study was conducted on 300 people aged 60 and above, including 149 patients in a hospital setting and 151 people cared for in a primary care setting in their place of residence.

The average score on the IADL scale was high (23.09) for the study group, while GDS scores indicated no depression in 203 subject and risk of depression or depression of increasing severity in 97 subjects. The entire study group represented a sufficient level of instrumental activity of daily living and good mental status.

The authors' own research results indicated that in the study group of elderly people, frailty diagnostic criteria according to the SHARE-FI questionnaire were met by $59.1 \%$ of hospitalized patients, while $26.8 \%$ were considered pre-frail. In turn, in the group of elderly people in a primary care setting, frailty was diagnosed in $12 \%$, whereas every third elderly person (33.7\%) appeared to be in the risk group. As indicated by the authors' own research results, the incidence of high frailty syndrome among hospitalized elderly patients suggests the need for introducing routine frailty assessment as part of the Comprehensive Geriatric Assessment (CGA) process [29].

According to Santos Eggimman et al., the proportion of frailty or prefrailty was generally higher in southern Europe than in northern Europe. In the middle-aged population, 4.1\% (95\% CI 3.4-4.7) were frail, and $37.4 \%(35.8-39.1)$ were prefrail [17].

The study by Bieniek et al., carried out on a group of 500 elderly patients hospitalized in a geriatrics department, indicates that the incidence of frailty increases with age. The syndrome was recognized in over $50 \%$ of the examined patients. The authors point to the necessity of routine frailty assessment as part of CGA [30].

In turn, the authors' own research results received from the group of patients in a primary care setting indicated the need for introducing optimal and useful scales for early frailty problem recognition and their implementation in everyday practice.

The conducted analysis confirmed SHARE-FI reliability as a tool to assess the risk of frailty syndrome of elderly people (above 60 years of age) in the Polish population. The scale reliability, evaluated based on Cronbach's alpha internal consistency measure, was 0.79 (primary care patients 0.79 and hospital patients 0.80 , respectively). This result demonstrated an acceptable level of internal consistency.

The study by Dorner et al. concerning a relationship between nutritional status and presence of frailty examined with SHARE-FI demonstrated overlaps in three dimensions: nutrition problems, mobility problems and anthropometric items, with moderate to high Cronbach's alpha - 0.670, 0.834 and 0.946, respectively [31].

In our study, we received satisfactory SHARE-FI scale reliability, allowing for its recommendation as a screening tool in primary care and hospital patients over 60 years of age and of both genders. Cronbach's alpha was 0.79 , indicating an acceptable level of reliability, both for group and individual comparison.

\section{Conclusions}

The research results indicated that the Polish version of the SHARE-FI questionnaire is characterized by high internal consistency and reliability and can be recommended for the screening assessment frailty risk among people above 60 years of age for both genders and in primary care and hospital settings.

Acknowledgements. We would like to thank all the participants of the test and the nurses who were willing to support us with the research.

Source of funding: This work was founded by a statutory grant for research topic no. 941/2016.

Conflict of interest: The authors declare no conflict of interests.

\section{References}

1. Kojima G. Frailty as a predictor of hospitalization among community-dwelling older people: a systematic review and meta-analysis. J Epidemiol Community Health 2016; 70(7): 722-729.

2. Hartgerink JM, Cramm JM, Bakker TJ, et al. The importance of multidisciplinary teamwork and team climate for relational coordination among teams delivering care to older patients. J Adv Nurs 2014; 70(4): 791-799.

3. Mattos IE, Carmo CN, Santiago LM, et al. Factors associated with functional incapacity in elders living in long stay institutions in Brazil: a cross-sectional study. BMC Geriatr 2014; 15(14): 47, doi: 10.1186/1471-2318-14-47.

4. Chang SF, Lin PL. Frail phenotype and mortality prediction: a systematic review and meta-analysis of prospective cohort studies. Int J Nurs Stud 2015; 52(8): 1362-1374.

5. Bagshaw SM, Stelfox HT, Johnson JA, et al. Long-term association between frailty and health-related quality of life among survivors of critical illness: a prospective multicenter cohort study. Crit Care Med 2015; 43(5): 973-982.

6. Mello Ade C, Engstrom EM, Alves LC. Health-related and socio-demographic factors associated with frailty in the elderly: a systematic literature review. Cad Saude Publica 2014; 30(6): 1143-1168.

7. Ní Mhaoláin AM, Fan CW, Romero-Ortuno R, et al. Depression: a modifiable factor in fearful older fallers transitioning to frailty? Int J Geriatr Psychiatry 2012; 27(7): 727-733. 
8. Kojima G, Taniguchi Y, lliffe S, et al. Frailty as a predictor of Alzheimer disease, vascular dementia, and all dementia among community-dwelling older people: a systematic review and meta-analysis. J Am Med Dir Assoc 2016; 17(10): 881-888, doi: 10.1016/j. jamda.2016.05.013.

9. Sutton JL, Gould RL, Daley S, et al. Psychometric properties of multicomponent tools designed to assess frailty in older adults: a systematic review. BMC Geriatr 2016; 16: 55, doi: 10.1186/s12877-016-0225-2.

10. Ruan Q, Yu Z, Chen M, et al. Cognitive frailty, a novel target for the prevention of elderly dependency. Ageing Res Rev 2015; 20: 1-10, doi: 10.1016/j.arr.2014.12.004.

11. Cano A, Kurpas D, Bujnowska-Fedak MM, et al. FOCUS: Frailty Management Optimisation through EIPAHA Commitments and Utilisation of Stakeholders' Input - an innovative European Project in elderly care. Fam Med Prim Care Rev 2016; 18(3): 373-376.

12. Freitag S, Schmidt S. Prevention of frailty through narrative intervention. Soc Sci Med 2016; 160: 120-127, doi: 10.1016/j.socscimed.2016.05.023.

13. Ahmed N, Mandel R, Fain MJ. Frailty: an emerging geriatric syndrome. Am J Med 2007; 120(9): 748-753.

14. Ensrud KE, Ewing SK, Cawthon PM, et al. A comparison of frailty indexes for the prediction of falls, disability, fractures, and mortality in older men. J Am Geriatr Soc 2009; 57(3): 492-498.

15. Boyd CM, Xue QL, Simpson CF, et al. Frailty, hospitalization, and progression of disability in a cohort of disabled older women. Am J Med 2005; 118(11): 1225-1231.

16. Fried LP, Tangen CM, Walston J, et al. Frailty in older adults: evidence for a phenotype. J Gerontol A Biol Sci Med Sci 2001; 56(3): M146-M156.

17. Santos-Eggimann B, Cuenoud P, Spagnoli J, et al. Prevalence of frailty in middle-aged and older community-dwelling Europeans living in 10 countries. J Gerontol A Biol Sci Med Sci 2009; 64(6): 675-681.

18. De Lepeleire J, lliffe S, Mann E, et al. Frailty: an emerging concept for general practice. Br J Gen Pract 2009; 59(562): e177-e182.

19. Ortuno RR, Walsh CD, Lawlor BA, et al. A Frailty Instrument for primary care: findings from the Survey of Health, Ageing and Retirement in Europe (SHARE). BMC Geriatrics 2010: 57, doi: 10.1186/1471-2318-10-57.

20. Ortuno RR, O'Shea D, Kenny RA. The SHARE frailty instrument for primary care predicts incident disability in a European populationbased sample. Qual Prim Care 2011; 19(5): 301-309.

21. Ortuno RR. The Frailty Instrument for primary care of the Survey of Health, Ageing and Retirement in Europe predicts mortality similarly to a frailty index based on comprehensive geriatric assessment. Geriatr Gerontol Int 2013; 13(2): 497-504.

22. Ortuno R, Soraghan C.A. Frailty Instrument for primary care for those aged 75 years or more: findings from the Survey of Health, Ageing and Retirement in Europe, a longitudinal population-based cohort study (SHARE-FI75+). BMJ Open 2014; 4(12): e006645, doi: 10.1136/ bmjopen-2014-006645.

23. Yesavage JA, Brink T, Lom O, et al. Development and validation of a geriatric depression screening scale: a preliminary report. J Psychiatr Res 1983; 17(1): 37-49.

24. Sheikh JI, Yesavage JA. Geriatric Depression Scale (GDS): recent evidence and development of a shorter version. Clin Gerontol 1986; 5(1/2): 165-173.

25. Lawton MP, Brody EM. Assessment of older people: self - maintaining and instrumental activities of daily living. The Gerontologist 1969; 9(3): 179-186.

26. Rockwood K, Song X, MacKnight Ch, et al. A global clinical measure of fitness and frailty in elderly people. CMAJ 2005; 173(5): 489-495.

27. Życzkowska J, Grądalski T. FS - what should to know the oncologist? Onkol Pract Clin 2010; 6(2): 79-84.

28. Rockwood K, Abeysundera MJ, Mitnitski A. How should we grade frailty in nursing home patients? J Am Med Dir Assoc 2007; 8(9): 595-603.

29. Rubenstein LZ. An overview of comprehensive geriatric assessment. In: Rubenstein LZ, Wieland D, Bernabei R, eds. Geriatric assessment technology: the state of the art. Milan: Editrice Kurtis; 1995: 1-9.

30. Bieniek J, Wilczyński K, Szewieczek J. Fried frailty phenotype assessment components as applied to geriatric inpatients. Clin Interv Aging 2016; 11: 453-459, doi: 10.2147/CIA.S101369.

31. Dorner TE, Luger E, Tschinderle J, et al. Association between nutritional status (MNA ${ }^{\circledR}$-SF) and frailty (SHARE-FI) in acute hospitalised elderly patients. J Nutr Health Aging 2014; 18(3): 264-269.

Tables: 3

Figures: 1

References: 31

Received: 07.04.2017

Reviewed: 25.04.2017

Accepted: 11.07.2017

Address for correspondence:

Marta Muszalik, RN, MD, PhD, Assoc. Prof.

Katedra i Klinika Geriatrii CM UMK

ul. M. Curie Skłodowskiej 9

85-094 Bydgoszcz

Polska

Tel.: +48 52 585-49-00

E-mail: muszalik@cm.umk.pl 